\title{
Imaging System For Measuring Macromolecule Crystal Growth Rates in Microgravity
}

\author{
Eric L. Corder,* Jeri Briscoe, ** \\ * Engineering Directorate, Marshall Space Flight Center-NASA, ED12, Huntsville, AL 35812 \\ ** Engineering Directorate, Marshall Space Flight Center-NASA, ED12, Huntsville, AL 35812
}

In order to determine how macromolecule crystal quality improvement in microgravity is related to crystal growth characteristics, a team of scientists and engineers at NASA's Marshal Space Flight Center (MSFC) developed flight hardware capable of measuring the crystal growth rates of a population of crystals growing under the same conditions. As crystal growth rate is defined as the change or delta in a defined dimension or length $(L)$ of crystal over time, the hardware was named Delta-L. Delta-L consists of three sub assemblies: a fluid unit including a temperature-controlled growth cell, an imaging unit, and a control unit (consisting of a Data Acquisition and Control Unit (DACU), and a thermal control unit). Delta- $\mathrm{L}$ will be used in connection with the Glovebox Integrated Microgravity Isolation Technology (g-LIMIT) inside the Microgravity Science Glovebox (MSG), onboard the International Space Station. This paper will describe the Delta-L imaging system.

The Delta-L imaging system was designed to locate, resolve, and capture images of up to 10 individual crystals ranging in size from 10 to $500 \mu \mathrm{m}$ with a point-to-point accuracy of $\pm 2.0 \mu \mathrm{m}$ within a quartz growth cell observation area of $20 \mathrm{~mm} \times 10 \mathrm{~mm} \times 1 \mathrm{~mm}$. The optical imaging system is comprised of a video microscope camera mounted on computer controlled translation stages (Fig. 1). The 3-axis translation stages (SD Instruments Inc., Grants Pass, OR) and control units (MicroMo Electronics, Lakeland, FL) provide crewmembers the ability to search throughout the growth cell observation area for crystals forming in size of approximately $10 \mu \mathrm{m}$. Once the crewmember has selected ten crystals of interest, the growth of these crystals is tracked until the size reaches approximately $500 \mu \mathrm{m}$. In order to resolve these crystals an optical system with a magnification of $10 \mathrm{X}$ was designed. A black and white NTSC camera (PULNiX, Sunnyvale, CA) was utilized with a 20X microscope objective (Mitutoyo, Aurora, $\mathrm{IL}$ ) and a $0.5 \mathrm{X}$ custom designed relay lens with an inline light (Creative Devices, Neshanic Station, NJ) to meet the magnification requirement. The design allows a $500 \mu \mathrm{m}$ crystal to be viewed in the vertical dimension on a standard NTSC monitor (4:3 aspect ratio). Images of the 10 crystals are collected periodically and stored in sets by the DACU (Matrox, Dorval, Canada).

The lighting for the Delta-L imaging system must be capable of illuminating the front face of a nearly transparent 3-D crystal structure growing inside a clear fluid and attached to the inside front wall of a quartz growth cell (NSG Precision Cells, Farmingdale, NJ). The most appropriate lighting design for enhancing the edges of this type of crystal structure is collimated backlighting. However, the growth cell is attached to a thermal electric cooler (TEC), which provides a temperaturecontrolled environment to enhance crystal growth. The TEC placement does not allow lighting to be transmitted through the back of the growth cell toward the video camera. Part of the solution to this problem was to coat the backside of the growth cell with aluminum prior to attaching it to the TEC. This enables the back of the growth cell to act as a mirror (Fig. 2). To complete the lighting solution, 
a green LED was folded into the optical axis at the back focal length of the microscope objective. This enabled the light to be concentrated on the front face of the crystal structure located at the front focal length $(30 \mathrm{~mm})$ of the microscope objective. Utilizing the microscope objective to transmit a focused on-axis front light enables light reflected from the mirrored surface of the growth cell to penetrate the crystal structure and produce a high contrast interface at the front face (Figs. $3 \& 4$ ). Not only did this lighting technique allow the front surface shape of the crystal structure to be measured, it also produced a gradual change in brightness on the sides of the crystal based on the slope of it sides. Images collected of each of the 10 crystals from the time of their growth from 50 $\mu \mathrm{m}$ to $500 \mu \mathrm{m}$ are analyzed and growth rate dispersion is calculated for the crystal set [1][2].

\section{References}

[1] D. A. H. Cunningham et al., Advances in Industrial Crystallization, Butterworth-Heinemann, (1991) 105-127.

[2] The Delta-L imaging design team includes Paul Julino of Morgan Research, Robert Copper and Dyana Beabout of NASA Marshall Space Flight Center.

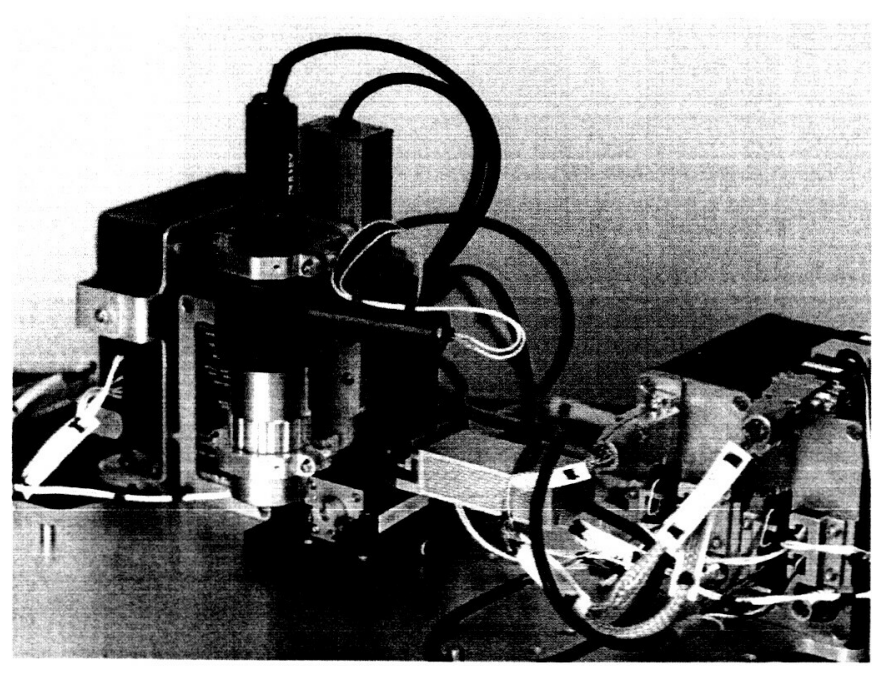

FIG. 1. Flight Optical Imaging System

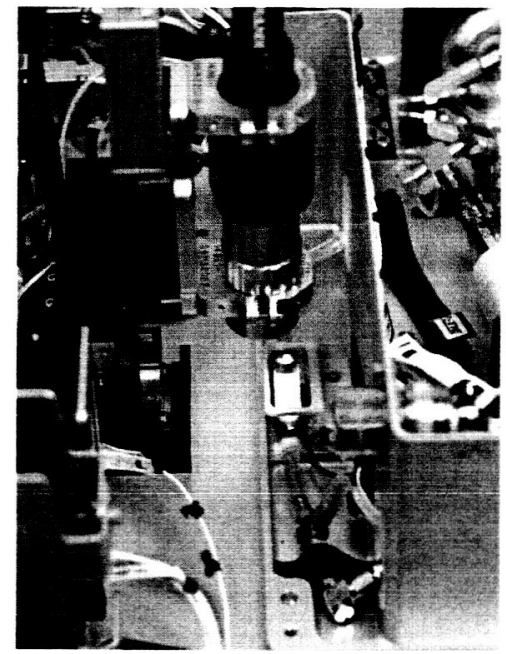

FIG. 2. Flight Imaging Unit viewing crystal growth cell

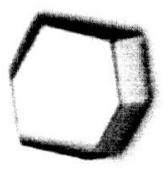

FIG. 3. $\approx 150 \mu \mathrm{m}$ Crystal Image

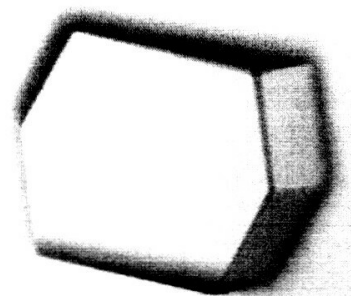

FIG. 4. $\approx 350 \mu \mathrm{m}$ Crystal Image 